\title{
Early Activation of Inflammation and Clotting in the Preterm Lamb with Neonatal RDS: Comparison of Conventional Ventilation and High Frequency Oscillatory Ventilation
}

\author{
ANNEKE S. JAARSMA, MARGRIETHE A. BRAAKSMA, WIL B. GEVEN, \\ WIM VAN OEVEREN, AND SIDARTO BAMBANG OETOMO \\ Department of Pediatrics, Division of Neonatology, University Hospital Groningen [A.S.J., M.A.B., \\ W.B.G., S.B.O.], and Department of Biomedical Engineering, Division of Biocompatibility, University of \\ Groningen [W.V.O.], The Netherlands.
}

\begin{abstract}
In neonatal respiratory distress syndrome activation of inflammation and clotting is demonstrated. High frequency oscillatory ventilation (HFOV) is considered to be less damaging to the human preterm lung, resulting in less activation of inflammation and clotting compared with conventional ventilation (CV). To assess the sequence of events of activation of inflammation and clotting and to compare the impact of HFOV to CV, we ventilated preterm lambs delivered by cesarean section at $132 \mathrm{~d}$ gestational age (term $145 \mathrm{~d})$ for $8 \mathrm{~h}$ by $\mathrm{CV}(n=10)$ or $\operatorname{HFOV}(n=11)$. Fifteen minutes after birth and at 2-h intervals thereafter blood samples, from umbilical catheters, were analyzed for AP50 (complement activation), number of polymorphonuclear leukocytes, $\beta$-glucuronidase, platelet function, activated partial thromboplastin time, thrombin time and thrombin inhibition, and bronchoalveolar lavage fluid was analyzed for elastase, thrombin and protein. We found complement activation, low number of polymorphonuclear leukocytes and high levels of $\beta$-glucuronidase already at $15 \mathrm{~min}$ after birth. Within 2 to $4 \mathrm{~h}$ after birth platelet function deteriorated, activated partial thromboplastin time prolonged, and thrombin inhibition decreased. Activation of inflammation and clotting in the lungs was dem-
\end{abstract}

\section{ABSTRACT}

onstrated by increased levels of elastase and thrombin in bronchoalveolar lavage fluid. In the HFOV group, AP50 remained significantly higher than in the CV group, reflecting less complement activation, and platelet function analysis remained significantly lower, reflecting better platelet function. We conclude that systemic activation of inflammation can be found in the ventilated preterm lamb with respiratory distress syndrome within 15 min after birth. Afterward, or due to activation of inflammation, clotting is activated. HFOV possibly attenuates activation of inflammation. (Pediatr Res 50: 650-657, 2001)

\section{Abbreviations}

RDS, neonatal respiratory distress syndrome

BALF, bronchoalveolar lavage fluid

PMNs, polymorphonuclear leukocytes

HFOV, high frequency oscillatory ventilation

$\mathbf{C V}$, conventional ventilation

$\mathbf{P a O}_{2}$, partial pressure of arterial $\mathrm{O}_{2}$

$\mathbf{P a C O}_{2}$, partial pressure of arterial $\mathrm{CO}_{2}$

APTT, activated partial thromboplastin time
Neonatal Respiratory Distress Syndrome (RDS) occurs in preterm infants, due to structural immaturity of the lungs and immaturity of the pulmonary surfactant metabolism, resulting in surfactant deficiency and surfactant dysfunction. Apart from these factors there is increasing evidence that inflammation and clotting contribute to the progression of this disease.

Already in 1983, Merritt et al. described that $3 \mathrm{~d}$ after birth an enhanced inflammatory response could be found in bronchoalveolar lavage fluid (BALF) of infants developing bron-

Received March 15, 2000; February 13, 2001.

Correspondence: A.S. Jaarsma, MD, Department of Pediatrics, University Hospital Groningen, P.O. box 30.001, 9700 RB Groningen, The Netherlands; e-mail: a.s.jaarsma@bkk.azg.nl chopulmonary dysplasia after RDS (1). This observation was confirmed by other investigators. Murch et al. found in the lungs of infants who died because of acute RDS, excessive polymorphonuclear leukocytes (PMNs) and alveolar macrophages (2). Other authors found PMNs, alveolar macrophages and pro-inflammatory cytokines in BALF, reflecting local activation of inflammation in the lungs of preterm infants with RDS, prone to development of bronchopulmonary dysplasia (1, $3,4-8)$.

However, as is known for ARDS and shock in adults, not only local activation of inflammation could be expected, but also systemic activation of inflammation (9). Recently Brus et al. reported that in RDS and chronic lung disease in preterm 
infants, systemic activation of inflammation could be found also $(10,11)$.

Clotting also plays an important role in the pathogenesis of RDS. As is known from histopathologic studies, fibrin is a major part of hyaline membranes and therefore hyaline membranes can be regarded as locally produced clots in RDS (12, 13). Schmidt described that intravascular thrombin-antithrombin III complex formation was related to disease severity in RDS (14). A similar observation was described by Brus et al. who investigated the systemic activation of clotting and fibrinolysis. A correlation was found between activation of clotting and the severity of protein leakage in the lungs of preterm rabbits as well as between the activation of clotting and the clinical severity of RDS in preterm infants $(15,16)$. These studies strongly suggest that apart from inflammation, local as well as systemic activation of clotting is involved in the pathogenesis of RDS.

In the light of the complex interaction between the inflammatory reaction and clotting, this finding is not surprising. However, the sequence of events in terms of local and systemic activation of inflammation and clotting remains to be elucidated.

It is suggested that both inflammation and clotting can be activated as the result of mechanical ventilation on the preterm lung (17). Since preterm lambs could not survive without mechanical ventilation, we decided to introduce high frequency oscillatory ventilation (HFOV) in one of the groups of lambs, being a mode of ventilation that is considered to be less damaging to the human preterm lung $(18,19)$.

Hence the aim of the study was to investigate the sequence of events in local as well as systemic activation of inflammation and clotting in the preterm lamb with RDS and to compare the impact of different ventilatory modes (conventional ventilation $(\mathrm{CV})$ versus $\mathrm{HFOV})$.

\section{METHODS AND MATERIALS}

The experiments were performed in the central animal laboratory of the University of Groningen, under approved animal care protocols of the University of Groningen with concern for animal welfare. We studied 21 preterm lambs of $132 \mathrm{~d}$ gestational age (term $145 \mathrm{~d}$ ). We chose lambs of $132 \mathrm{~d}$ because it is known that at this gestational age, lambs suffer from RDS (20). We saw in a pilot study that lambs that were not ventilated, died with the clinical picture of RDS: tachypnea, dyspnea, grunting, cyanosis, respiratory acidosis, and ultimately respiratory failure.

Treatment of the animals. The ewes were sedated with Sevoflurane inhalation anesthesia and a cesarean section through a lateral incision was performed under local anesthesia with s.c. prilocaine. Ewes were not selected for twin pregnancy. Before starting each single experiment we randomized the firstborn lamb to CV or HFOV by coin method. If there was a twin pregnancy, the second lamb underwent the same procedure as the first born lamb, but was assigned to the alternate ventilatory mode.

The lambs were delivered and, before clamping and cutting of the umbilical cord, they were intubated orotracheally with a cuffed 5.5-mm ID tube and the airways were suctioned once. After delivery the lambs were dried and weighed and mechanically ventilated immediately with a time-cycled pressurelimited infant ventilator (Babylog 8000, Dräger, Lübeck, Germany) for $\mathrm{CV}$ or a High Frequency Oscillatory Ventilator (Sensor Medics 3100, Yorba Linda, CA, U.S.A.) for HFOV. Initial ventilator settings of $\mathrm{CV}$ were: frequency $60 / \mathrm{min}$, inspiratory time equal to expiratory time at $0.5 \mathrm{~s}$, gas flow 8 $\mathrm{L} / \mathrm{min}$. Peak inspiratory pressure was initially set at $20 \mathrm{~cm} \mathrm{H}_{2} \mathrm{O}$ and was adjusted immediately after the start of ventilation to achieve a tidal volume of 5-8 $\mathrm{mL} / \mathrm{kg}$ and an expiratory minute volume of about $250 \mathrm{~mL} / \mathrm{kg} / \mathrm{min}$. Positive end-expiratory pressure was set at $4 \mathrm{~cm} \mathrm{H}_{2} \mathrm{O}$. Fractional concentration of inspired $\mathrm{O}_{2}$ was set at 0.4 and adjusted to maintain transcutaneous $\mathrm{O}_{2}$ saturation between 92 and $95 \%$ (measured with Pulseoximeter Nellcor, Hayward, CA, U.S.A.) and $\mathrm{PaO}_{2}$ between 6.0 and 8.0 $\mathrm{kPa}$. During the study peak inspiratory pressure was adjusted to achieve $\mathrm{PaCO}_{2}$ values of 5.5 to $7.0 \mathrm{kPa}$ provided that $\mathrm{pH}$ was $>7.25$.

Initial ventilator settings of HFOV were: frequency $10 \mathrm{~Hz}$, inspiratory time 0.33 , gas flow $20 \mathrm{~L} / \mathrm{min}$. Mean airway pressure was initially set at $14 \mathrm{~cm} \mathrm{H}_{2} \mathrm{O}$. Fractional concentration of inspired $\mathrm{O}_{2}$ was set at 0.4 and after stabilization, we searched for optimal distending pressure, aiming at transcutaneous $\mathrm{O}_{2}$ saturation between 92 and $95 \%$ and $\mathrm{PaO}_{2}$ between 6.0 and 8.0 $\mathrm{kPa}$ with the lowest possible fractional concentration of inspired $\mathrm{O}_{2}$ preferably below 0.3 . Amplitude was initially adjusted according to oscillatory movements of the chest wall and afterward adjusted to achieve $\mathrm{PaCO}_{2}$ values of 5.5 to $7.0 \mathrm{kPa}$ provided that $\mathrm{pH}$ was $>7.25$.

In all lambs, an arterial catheter was advanced in the descending aorta through an umbilical artery and was used for blood sampling and measurement of blood pressure. Patency was maintained by continuous infusion of a saline solution $0.9 \%$ at a rate of $2.0 \mathrm{~mL} / \mathrm{h}$. During the study blood pressure was maintained in the normal range (mean $40 \mathrm{~mm} \mathrm{Hg}$ ). In case of hypotension, a $10 \mathrm{~mL} / \mathrm{kg}$ bolus of saline solution $0.9 \%$ was administered i.v. A venous catheter was advanced in the vena cava inferior through the umbilical vein and was used for the administration of fluids $(3 \mathrm{~mL} / \mathrm{kg} / \mathrm{h})$, glucose $(5 \mathrm{mg} / \mathrm{kg} / \mathrm{min})$ and sodium pentobarbital. Serum glucose levels were measured two-hourly and were kept in the normal range (4-9 $\mathrm{mmol} / \mathrm{L}$ ) by adjusting the amount of glucose that was infused. Body temperature was measured continuously by a rectal thermometer. Body temperature was kept at $38^{\circ} \mathrm{C}$ by using preheated mattress and blankets. Sodium pentobarbital (Nembutal $^{\mathbb{B}}$ ) was given for sedation, initially $15 \mathrm{mg} / \mathrm{kg} / \mathrm{h}$, dosage was adjusted according to activity, blood pressure, and heart rate of the lamb.

Blood sampling. Blood samples of $0.4 \mathrm{~mL}$ were obtained hourly for blood-gas analysis in heparinized syringes. Blood samples of $5.25 \mathrm{~mL}$ were obtained immediately after inserting catheters (15 min after birth) and 2,4,6, and $8 \mathrm{~h}$ after initiating ventilation. Five milliliters of this blood was anticoagulated with citrate $0.31 \%$ immediately and placed on ice. It was used to determine AP50, $\beta$-glucuronidase, platelet function, activated partial thromboplastin time (aPTT), thrombin time, thrombin inhibition, and urea. The other $0.25 \mathrm{~mL}$ blood was 
anticoagulated with EDTA $0.01 \mathrm{M}$ and was used to determine total leukocyte count, and to make blood smears for differential cell counts, especially to determine the number of PMNs.

Bronchoalveolar lavage. Immediately after intubation the lambs' airways were suctioned. Bronchoalveolar lavage was performed 2, 4, 6, $8 \mathrm{~h}$ after intubation by injecting $1 \mathrm{~mL} / \mathrm{kg}$ saline solution $0.9 \%$ intratracheally with immediate aspiration, this was repeated three times. To exclude effects of bronchoalveolar lavage on activation of inflammation and coagulation, we alternated bronchoalveolar lavage with dry suctioning in successive lambs. In aspirated material (from now on it is called BALF) elastase, thrombin and protein concentrations, corrected for urea, were determined. Among the lambs in whom bronchoalveolar lavage was performed and lambs in whom dry suctioning was performed, no differences in either ventilatory parameters, nor laboratory results were found, so these lambs are combined for further analysis.

After $8 \mathrm{~h}$ the study was ended, the lamb was euthanized with an overdose sodium pentobarbital, and a thoracotomy was performed to remove the lungs.

Processing of the samples. The heparinized blood was used for blood gas analysis to assess $\mathrm{PaO}_{2}, \mathrm{PaCO}_{2}$, and $\mathrm{pH}$, using an ABL 330 blood gas analyzer (Radiometer Co, Copenhagen, Denmark) and to assess $\mathrm{Hb}$ and $\mathrm{SaO}_{2}$ using an OSM-3 analyzer (Radiometer Co, Copenhagen, Denmark). $0.8 \mathrm{~mL}$ of the citrated blood was used directly to determine platelet function, using the Platelet Function Analyzer (DADE International Inc., Miami, U.S.A.). $3.7 \mathrm{~mL}$ of the blood was centrifuged at 1100 $\mathrm{rpm}$ for $10 \mathrm{~min}$ and the plasma was stored at $-80^{\circ} \mathrm{C}$ until AP50, $\beta$-glucuronidase aPTT, thrombin time, thrombin inhibition, and urea were determined. The BALF and lung aspirate samples were centrifuged at $1100 \mathrm{rpm}$ and the supernatant was stored at $-80^{\circ} \mathrm{C}$ until further processing took place.

Assays. AP50 titer (the alternative pathway hemolytic activity assay as a measure of activation of alternative route of complement system) is the plasma dilution that causes $50 \%$ lysis of nonsensitized rabbit erythrocytes in a standard suspension after prevention of activation of the classical route with EGTA and $\mathrm{Mg}^{2+}$ (21). A reduced value indicates more complement consumption due to activation.

Determination of total leukocyte count from EDTA blood was performed microscopically using a chamber after a gentian violet staining. To determine differential cell counts, Giemsa stained smears were used. The total number of PMNs was calculated from these two measurements.

$\beta$-glucuronidase (released from PMNs after activation) was measured according to Baehner (22). For this purpose $25 \mu \mathrm{L}$ lamb plasma was incubated with $25 \mu \mathrm{L}$ p-nitrophenyl $\beta$-Dglocopyranosid uric acid solution for $2 \mathrm{~h}$ at $37^{\circ} \mathrm{C}$. The conversion of the substrate by $\beta$-glucuronidase was determined at 405 $\mathrm{nm}$ using a microplate reader (Microplate reader $3550 \mathrm{UV}$, Bio-Rad, Richmond CA, U.S.A.). After determination it was corrected for the number of PMNs. Specificity of substrate used in the assay was tested by adding a cocktail of inhibitors (C1esterase inhibitor, hirudin, trypsin, and aprotinin), which inhibit complement, thrombin, trypsin, chymotrypsin, plasmin, kallikrein, and activated protein $\mathrm{C}$, to the test substance. We found no reduction of $\beta$-glucuronidase activity. So we concluded that the specificity of the substrate was good.

Platelet function analysis was carried out using the Platelet Function Analyzer, using PFA-100 collagen/ADP test cartridges (DADE International Inc., Miami, U.S.A.). This instrument reproduces the exposure to an injured vessel. In the Platelet Function Analyzer, platelets are maximally stimulated to form a clot. Increased value of platelet function analysis is a sign of increased clotting because it takes longer to form a clot when the number of platelets is diminished or when the present platelets are "exhausted" due to activated clotting in vivo. No references are known for the use of Platelet Function Analyzer in the analysis of the function of sheep platelets, but the reliability of the Platelet Function Analyzer has been described by Heilmann et al. (23).

The aPTT (a screening test for abnormalities in the intrinsic clotting system, especially factors VIII, IX, XI, XII, X, and II, and prekallikrein, high molecular weight kininogen and fibrinogen) was measured by adding $100 \mu \mathrm{L}$ aPTT reagent (Sigma Chemical Co. Diagnostics, St. Louis, U.S.A.) and $100 \mu \mathrm{L}$ calcium chloride to lamb plasma and recording clotting time. Thrombin time, a screening test for the common pathway of the clotting system and especially measuring polymerization of fibrinogen to fibrin was measured by adding $100 \mu \mathrm{L}$ thrombin time reagent (Sigma Chemical Co. Diagnostics, St. Louis, U.S.A.) to lamb plasma and recording clotting time.

Thrombin inhibition (a method to determine the amount of mainly antithrombin III in plasma) was measured by adding excess thrombin stock $(1.5 \mathrm{IU} / \mathrm{mL}$, Biopool, Ventura, CA, U.S.A.) to lamb plasma and measuring the remaining thrombin in the microplate reader at $405 \mathrm{~nm}$. A higher value indicates more remaining thrombin, reflecting a lower antithrombin III concentration in the tested plasma.

Urea was determined according to Marsh et al. (24).

Elastase in BALF (a measurement of local activation of PMNs in the lung) was measured by incubating BALF with elastase substrate (Omnilabo, Breda, The Netherlands) for three hours at $37^{\circ} \mathrm{C}$. Afterward the recorded OD value at 405 $\mathrm{nm}$ using the microplate reader was compared with a standard porcine pancreatic elastase. Specificity of substrate used in the assay was tested by adding a cocktail of inhibitors (C1esterase inhibitor, hirudin, trypsin and aprotinin), which inhibit complement, thrombin, trypsin, chymotrypsin, plasmin, kallikrein and activated protein $\mathrm{C}$, to the test substance. We found no reduction of elastase activity. So we concluded that the specificity of the substrate was good.

Thrombin in BALF (a measurement of local activation of the clotting system in the lung) was measured after adding a thrombin specific substrate (Chromogenix S2238, Mölndal, Sweden). A higher OD value at $405 \mathrm{~nm}$ using the microplate reader indicates a higher level of thrombin.

Protein in BALF (a measurement of capillary leakage due to local injury in the lung) was measured according to Lowry et al. (25). A higher OD value at $655 \mathrm{~nm}$ using the microplate reader indicates a higher protein level. To correct for dilution, values of elastase, thrombin, and protein are corrected for the measured urea in BALF. 


\section{DATA AND STATISTICAL ANALYSIS}

Ventilatory data are presented as mean and SD. Laboratory data are presented as mean and SEM. For statistical evaluation Wilcoxon signed-rank test was used to determine changes between the first sampling moment and successive moments. Student's $t$ test was used to determine differences between groups. Data were considered significant when $p<0.05$.

\section{RESULTS}

We studied 21 preterm lambs of $132 \mathrm{~d}$ gestational age. It was possible to ventilate preterm lambs of $132 \mathrm{~d}$ gestational age without surfactant, while maintaining adequate blood gas values. Ten lambs were ventilated with CV, 11 lambs were ventilated with HFOV. Ventilatory and oxygenation parameters are shown in Tables 1 and 2. Used fractional concentration of inspired $\mathrm{O}_{2}$, mean airway pressure, $\mathrm{PaO}_{2}, \mathrm{PaCO}_{2}$, oxygenation index and alveolar-arterial partial $\mathrm{O}_{2}$ tension difference did not differ significantly between the two groups. In the $\mathrm{CV}$ group 3 lambs received saline $(10,20$, and $30 \mathrm{~mL} / \mathrm{kg})$, in the HFOV group 2 lambs received saline (both $10 \mathrm{~mL} / \mathrm{kg}$ ). The mean (SD) total dose of Nembutal in the CV group was $20.3(11.8) \mathrm{mg} / \mathrm{kg}$ (total dose during the ventilation period), the mean (SD) total dose of Nembutal in the HFOV group was 30.2 (13.9) $\mathrm{mg} / \mathrm{kg}$. Those dosages were not statistically significant different (Student $t$ test, $p=0.1)$. Hb levels remained stable in both groups during the day. Between the groups were no statistically significant differences (CV: mean $\mathrm{Hb} 14.1 \mathrm{~g} / \mathrm{dL}$, SD 2.1, HFOV mean $13.3 \mathrm{~g} / \mathrm{dL}$, SD 2.4).

The Figs. 1, $A-F$ show laboratory data of blood analysis including AP50, total number of leukocytes, number of PMNs, $\beta$-glucuronidase (corrected for number of PMNs), platelet function analysis and aPTT in plasma. The Figs. $2, A-C$ show measurements in BALF of elastase, thrombin and protein corrected for urea in BALF. We detected the following changes in laboratory results concerning activation of inflammation and clotting in the preterm lambs with RDS.
AP50 (Fig. $1 A$ ) is already low within 15 min after birth (maternal sheep values of AP50 are about $200 \mathrm{IU} / \mathrm{mL}$ ). A significant increase is found at $2 \mathrm{~h}$ after birth compared with 15 min after birth only in the HFOV group $(p=0.04)$. Afterward AP50 remains stable like in the CV group. AP50 is statistically significant lower, reflecting more complement activation in the $\mathrm{CV}$ group than in the HFOV group at 6 and $8 \mathrm{~h}$ after birth $(p=0.04)$.

Total number of leukocytes (Fig. $1 B$ ) show a gradual increase during the period of ventilation. In the first $2 \mathrm{~h}$ after birth total number of leukocytes increases in the HFOV group whereas it decreases in the $\mathrm{CV}$ group. However the differences are not statistically significant.

PMNs (Fig. 1C) are low 15 min after birth in both groups (maternal sheep values of PMNs are 3500/ $\mu \mathrm{L}$ ) and increase gradually. A significant increase is already found at $2 \mathrm{~h}$ after birth compared with 15 min after birth in both groups (CV group $p=0.03$, HFOV group $p=0.04$ ) and remains significantly higher during the entire ventilation period compared with $15 \mathrm{~min}$ after birth. Between the groups there are no statistically significant differences.

$\beta$-glucuronidase (Fig. $1 D$ ) is high 15 min after birth in both groups (maternal sheep values of $\beta$-glucuronidase are 0.05 $\mathrm{OD} / \mathrm{PMN}$ ) and decreases gradually. A significant decrease is found at $2 \mathrm{~h}$ after birth in both groups (CV group $p=0.04$, HFOV group $p=0.05$ ) and remains significantly lower during the entire ventilation period compared with 15 min after birth. Between the groups there are no statistically significant differences.

Platelet function analysis (Fig. $1 E$ ) shows normal values during the first $4 \mathrm{~h}$ after birth in both groups. In the $\mathrm{CV}$ group after $4 \mathrm{~h}$ large differences are found in individual lambs. The values in the HFOV group remain stable. Platelet function value is significantly higher in the $\mathrm{CV}$ group than in the HFOV group at $8 \mathrm{~h}$ after birth $(p=0.01)$, reflecting better platelet function in the HFOV group than in the CV group.

Table 1. Ventilatory characteristics and blood gas analysis values for the two study groups at the moments blood samples for measurement of study parameters were taken

\begin{tabular}{|c|c|c|c|c|c|c|}
\hline & $\mathrm{t}=15 \min$ & $\mathrm{t}=2$ hours & $\mathrm{t}=4$ hours & $\mathrm{t}=6$ hours & $\mathrm{t}=8$ hours & \\
\hline \multicolumn{7}{|l|}{$\mathrm{CV}(n=10)$} \\
\hline Peak inspiratory pressure & $28.4 \pm 5.5$ & $27.9 \pm 5.1$ & $28.2 \pm 6.1$ & $28.1 \pm 7.0$ & $27.5 \pm 7.3$ & $\mathrm{~cm} \mathrm{H}_{2} \mathrm{O}$ \\
\hline Positive end-expiratory pressure & 4.1 & 4.1 & 4.1 & 4.1 & 4.1 & $\mathrm{~cm} \mathrm{H}_{2} \mathrm{O}$ \\
\hline Mean airway pressure & $14.0 \pm 2.0$ & $14.8 \pm 3.0$ & $14.3 \pm 2.1$ & $14.2 \pm 2.3$ & $14.3 \pm 2.8$ & $\mathrm{~cm} \mathrm{H}_{2} \mathrm{O}$ \\
\hline $\mathrm{PaO}_{2}$ & $22.8 \pm 16.9$ & $8.1 \pm 3.7$ & $7.9 \pm 2.9$ & $9.4 \pm 6.2$ & $7.6 \pm 2.1$ & $\mathrm{kPa}$ \\
\hline $\mathrm{PaCO}_{2}$ & $9.0 \pm 2.3$ & $6.0 \pm 1.6$ & $5.9 \pm 1.8$ & $6.4 \pm 1.8$ & $7.7 \pm 2.0$ & $\mathrm{kPa}$ \\
\hline $\operatorname{HFOV}(n=11)$ & $\mathrm{t}=15 \mathrm{~min}$ & $\mathrm{t}=2$ hours & $\mathrm{t}=4$ hours & $\mathrm{t}=6$ hours & $\mathrm{t}=8$ hours & \\
\hline Mean airway pressure & $17.5 \pm 4.1$ & $13.9 \pm 2.5$ & $13.9 \pm 3.3$ & $12.9 \pm 4.8$ & $13.8 \pm 3.7$ & $\mathrm{~cm} \mathrm{H}_{2} \mathrm{O}$ \\
\hline Fractional concentration of inspired $\mathrm{O}_{2}$ & $0.60 \pm 0.15$ & $0.36 \pm 0.15$ & $0.29 \pm 0.05$ & $0.30 \pm 0.06$ & $0.30 \pm 0.09$ & \\
\hline $\mathrm{PaO}_{2}$ & $34.1 \pm 16.7$ & $8.8 \pm 4.2$ & $8.8 \pm 5.0$ & $8.4 \pm 2.4$ & $7.3 \pm 1.9$ & $\mathrm{kPa}$ \\
\hline $\mathrm{PaCO}_{2}$ & $5.9 \pm 2.5$ & $7.6 \pm 7.4$ & $5.7 \pm 1.3$ & $5.4 \pm 1.2$ & $6.3 \pm 1.1$ & $\mathrm{kPa}$ \\
\hline $\mathrm{OI}^{*}$ & $5.5 \pm 3.7$ & $9.3 \pm 5.8$ & $7.7 \pm 3.6$ & $7.3 \pm 3.2$ & $8.1 \pm 4.0$ & $\mathrm{~mm} \mathrm{Hg}$ \\
\hline
\end{tabular}

Data are presented as mean $\pm \mathrm{SD}$.

* OI $=$ oxygenation index $=\left(\right.$ Mean airway pressure $\times$ fractional concentration of inspired $\left.\mathrm{O}_{2} / \mathrm{PaO}_{2}\right)$.

$\dagger \mathrm{AaDO}_{2}=$ alveolar-arterial partial $\mathrm{O}_{2}$ tension difference

$=$ fractional concentration of inspired $\mathrm{O}_{2}\left(\mathrm{P}_{\mathrm{atm}}-\mathrm{P}_{\mathrm{H} 2 \mathrm{O}}\right)-\mathrm{PaO}_{2}-\mathrm{PaCO}_{2}$. 
The aPTT (Fig. $1 F$ ) in plasma is normal 15 min after birth and increases significantly in the first $2 \mathrm{~h}$ after birth in both groups (CV group $p=0.04$, HFOV group $p=0.01)$. In the

A

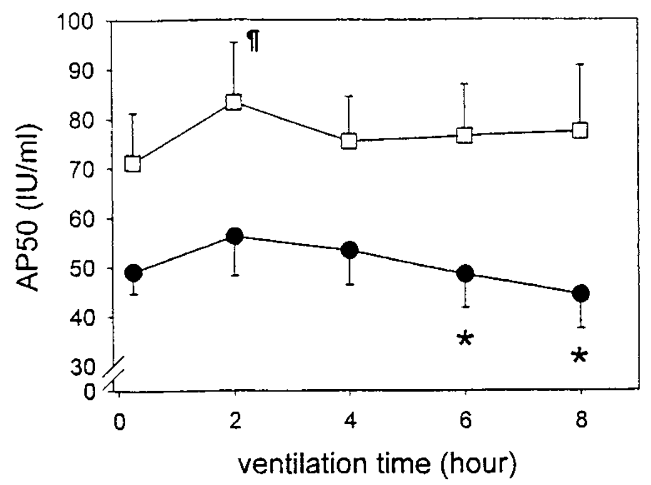

C

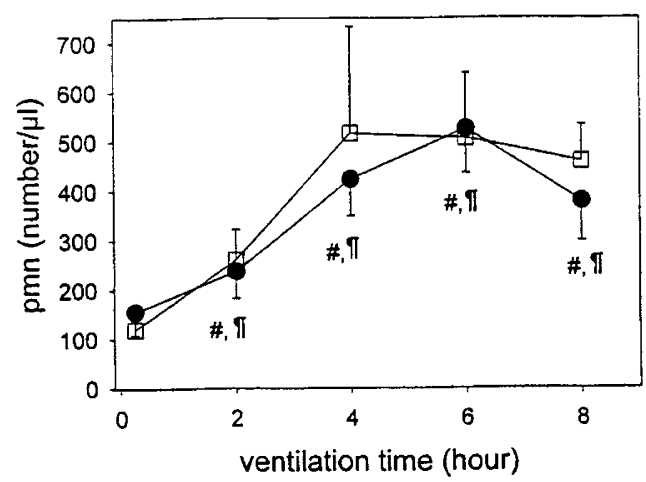

E

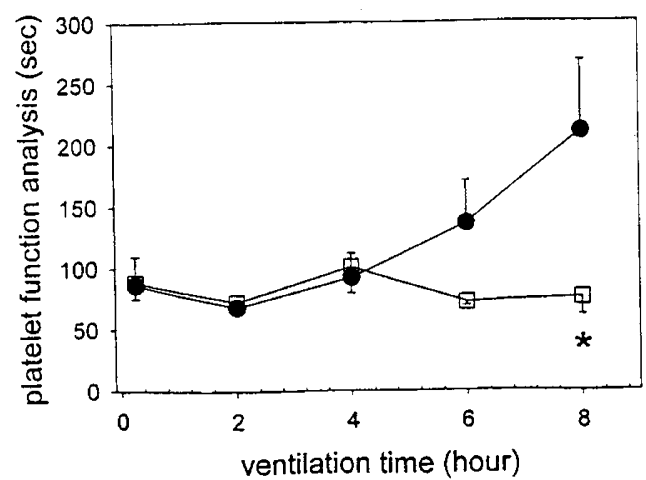

next $6 \mathrm{~h}$ aPTT decreases in both groups to values in the normal range, but in the HFOV group aPTT remains statistically significant higher during the entire ventilation period compared
B

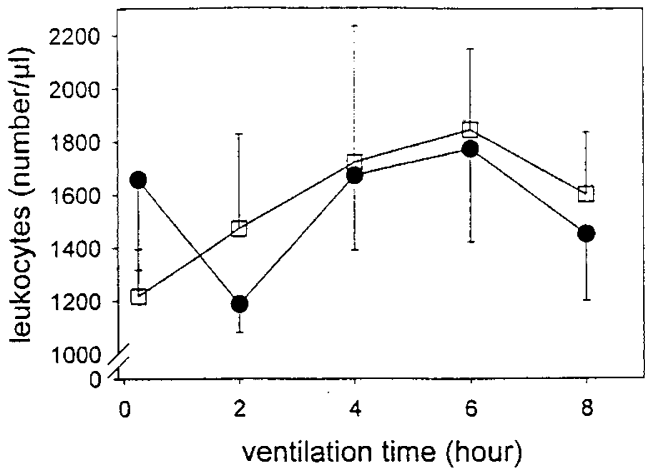

D

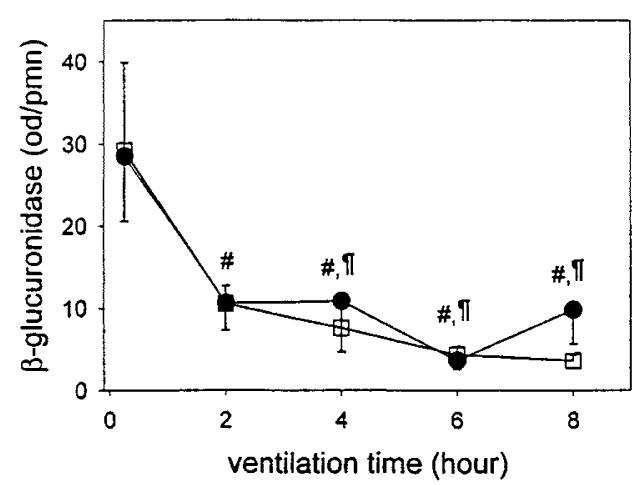

$\mathbf{F}$

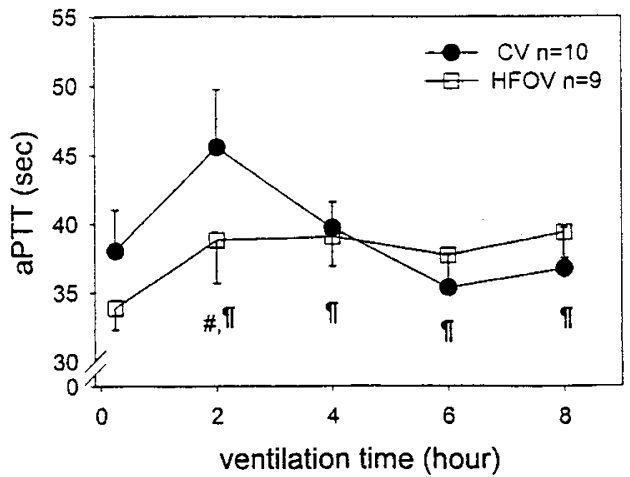

Figure 1. Concentrations of studied parameters in plasma of the CV $(--, n=10)$ and HFOV $(-\square-, n=11)$ ventilated preterm lambs at 15 min, $2,4,6$, and $8 \mathrm{~h}$ after birth. Data are presented as mean with SEM. \# $p<0.05$ compared with 15 min after birth within the CV group (Wilcoxon signed-rank test). $\mid p<$ 0.05 compared with 15 min after birth within the HFOV group (Wilcoxon signed-rank test). ${ }^{*} p<0.05$ for CV group $v s$ HFOV group (Student's $t$ test). (A) AP50 (IU/mL), $(B)$ Number of leukocytes (number $/ \mu \mathrm{L}),(C)$ Number of PMNs (number/ $\mu \mathrm{L}),(D)$-glucuronidase (corrected for number of PMNs, OD/PMN), $(E)$ platelet function analysis (seconds), $(F)$ aPTT (seconds, in the HFOV group 2 lambs are excluded because of immeasurable aPTT due to sampling error). 
with 15 min after birth. Between the groups there are no statistically significant differences.

Thrombin time (data not shown) in plasma remains in the normal range (mean values 11.2-15.3 s) during the entire ventilatory period in both groups. However within the HFOV group thrombin time increases significantly in the first $2 \mathrm{~h}$ after birth $(p=0.008)$ compared with 15 min after birth. Between the groups there are no statistically significant differences. Thrombin inhibition (data not shown) in plasma is lower (mean values $1.33-1.40 \mathrm{IU} / \mathrm{mL}$ in both groups) than maternal values $(1.45-1.5 \mathrm{IU} / \mathrm{mL}) 15 \mathrm{~min}$ after birth in both groups but remains in the initial range. Between the groups there are no statistically significant differences.

Elastase (Fig. 2A) in BALF is low just before birth $(\mathrm{t}=0)$ in both groups and increases significantly in the first $2 \mathrm{~h}$ after birth in both groups (CV group $p=0.01$, HFOV group $p=$ 0.006). Between the groups there are no statistically significant differences.

Thrombin activity (Fig. $2 B$ ) in BALF can be detected in both groups. In the HFOV group the value of thrombin is statistically significant higher at $4 \mathrm{~h}$ after birth compared with $\mathrm{t}=0$ $(p=0.004)$. Afterward it decreases to the same value as $t=0$. Between the groups there are no statistically significant differences.

Protein (Fig. 2C) in BALF shows the same tendency as elastase in BALF. Protein increases significantly in the first $2 \mathrm{~h}$ after birth in both groups (CV group $p=0.04$, HFOV group $p$ $=0.003$ ) and remains stable afterward. Between the groups there are no statistically significant differences. After analysis only $\mathrm{AaDO}_{2}$ at $\mathrm{t}=8 \mathrm{~h}$ was considered suitable for multiple regression analysis, because the other clinical values were not linearly and independently related to one of the laboratory values. Multiple regression analysis was performed for $\mathrm{AaDO}_{2}$ at $\mathrm{t}=8 \mathrm{~h}$ against all laboratory values and no significant correlations were found.

\section{DISCUSSION}

To investigate the sequence of events in local as well as systemic activation of inflammation and clotting in RDS, and to compare the impact of different ventilatory modes $(\mathrm{CV}$ versus $\mathrm{HFOV}$ ) on the activation of inflammation and clotting,
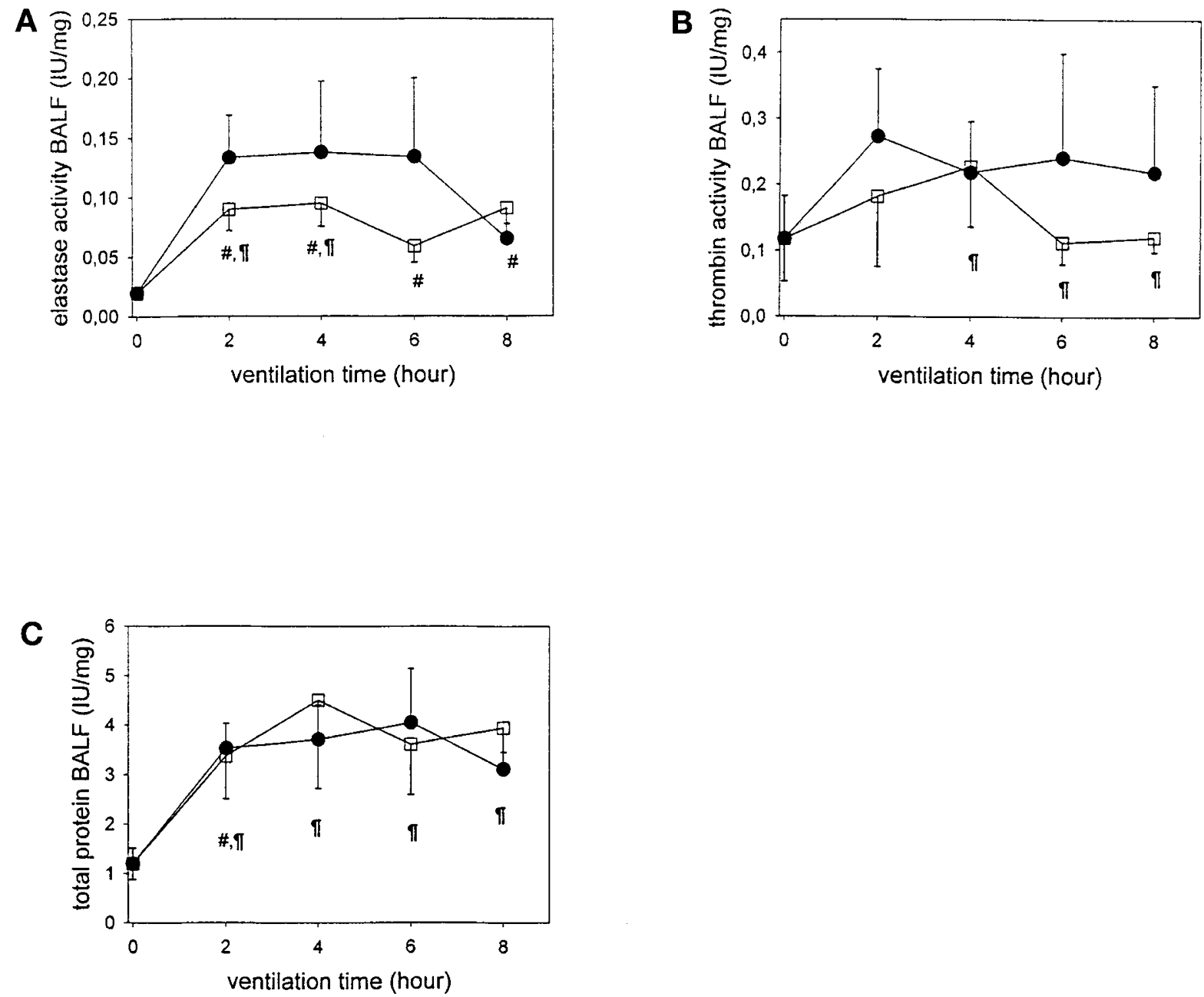

Figure 2. Concentrations of study parameters in BALF of the CV $(-\bullet-, n=10)$ and $\operatorname{HFOV}(-\square, n=11)$ ventilated preterm lambs at $\mathrm{t}=0$ (just before delivery and after intubation), 2, 4, 6, and $8 \mathrm{~h}$ after birth, corrected for urea concentrations in BALF. Data are presented as mean with SEM. \# $p<0.05$ compared with $\mathrm{t}=0$ within the $\mathrm{CV}$ group (Wilcoxon signed-rank test). $\uparrow p<0.05$ compared with $\mathrm{t}=0$ within the HFOV group (Wilcoxon signed-rank test). (A) elastase (IU/mg), (B) thrombin (IU/mg), (C) protein (IU/mg). 
we studied preterm lambs of $132 \mathrm{~d}$. We found activation of inflammation and clotting within 15 min after birth as well in the $\mathrm{CV}$ as in the HFOV group. HFOV possibly attenuates activation, but the results are not conclusive.

In a pilot study $(n=3)$ we ventilated the first born lamb with $\mathrm{CV}$ and used the second born lamb as a control. We found that these lambs suffered from such a severe RDS that without ventilation severe respiratory acidosis (mean $\mathrm{pH}$ : 6.76, mean $\mathrm{PaCO}_{2}: 16.8 \mathrm{kPa}$ ) developed and the lambs died within about $1 \mathrm{~h}$. Therefore the study protocol was re-designed to compare $\mathrm{CV}$ with HFOV. Adequate ventilation was possible without surfactant administration. Laboratory investigations concerning inflammation and clotting systemically (in plasma) as well as locally (in BALF) yielded adequate reproducible results. So we concluded that the preterm lamb of $132 \mathrm{~d}$ gestational age provide a good animal model to perform these studies.

We found complement activation, low number of PMNs and a high level of $\beta$-glucuronidase in plasma already 15 min after birth in both groups. These findings suggest that systemic inflammation has possibly occurred before or very soon after birth with subsequent pooling of PMNs among others in the lungs. Local activation of inflammation appears likely from the observation of increasing levels of elastase in BALF in the first $2 \mathrm{~h}$ after birth, remaining stable afterward. In this experiment we did not determine inflammatory cells in BALF, this is planned in the next experiment. However, elastase in BALF probably better reflects inflammatory reaction in alveoli than the number of inflammatory cells itself (26). The finding of activated inflammation is in accordance with previous studies, which showed systemic activation of inflammation in RDS (1, 5). A correlation between the severity of the inflammatory reaction (reflected by complement activation, decrease of PMN in the blood and release of $\beta$-glucuronidase) and the severity of protein leakage in the lungs was demonstrated in preterm rabbits (16). The same authors found a correlation between the severity of the inflammatory reaction and the clinical severity of RDS in preterm infants (27). Wagner et al. found complement activation in RDS patients which was more pronounced in patients with a poor response to surfactant administration (28). In addition, Carlton et al. described systemic effects of RDS: they found that PMNs disappeared from blood due to sequestration in the lungs of preterm lambs with RDS, causing increased vascular permeability (29). This effect could be attenuated in neutropenic lambs. Based on these studies, it was concluded that inflammation plays an important role in the pathogenesis of RDS as well as in the pathogenesis of the complications of RDS or bronchopulmonary dysplasia.

Clotting also plays an important role in the pathogenesis of RDS. We know fibrin deposits are found within the lung's intravascular, interstitial and intra-alveolar spaces in RDS (12). It is also known that platelets play an important role in inflammation. After platelet activation, platelet derived growth factor is released, P-selectin (necessary for PMN rolling on endothelial wall) is expressed and histamine is released, causing vasodilatation and increased vascular permeability (30). Therefore we studied activation of clotting in this study. Within 2 to $4 \mathrm{~h}$ after birth activation of clotting was found in both groups, reflected by deteriorating platelet function $4 \mathrm{~h}$ after birth, prolonging of aPTT within $2 \mathrm{~h}$, and lower thrombin inhibition in blood. The prolonged aPTT reflects factor VIII consumption due to clotting activation in plasma. Local activation of clotting appeared likely from the demonstration of thrombin activity in BALF, since thrombin is not found in normal lungs. However, because protein in BALF also increases in the first hours after birth, we cannot exclude the possibility that the thrombin activity in BALF is the result of capillary leakage. These findings suggest that systemic as well as local activation of clotting take place after birth. Because activation of clotting and platelet activation are found later than activation of inflammation, we consider activation of clotting is not causative in RDS, but is activated secondary to activation of inflammation.

HFOV is a mode of ventilation considered to be less damaging to human preterm lung than $\mathrm{CV}(18,19)$. Therefore, we compared CV to HFOV. There seemed to be less complement activation in the HFOV group, reflected by significantly less decreased AP50 levels than in the CV group after $6 \mathrm{~h}$ of ventilation. Both $\mathrm{CV}$ and $\mathrm{HFOV}$ ventilated animals showed a significant increase of elastase in BALF after 2 hour of ventilation compared with just before birth. Although elastase levels in BALF from lambs ventilated with CV were not statistically significant higher than elastase levels in BALF from lambs ventilated with $\mathrm{HFOV}$ the tendency toward higher elastase levels in CV ventilated lambs is in accordance with the results in blood, suggesting a protective effect of HFOV. Similar results were described by other investigators. Imai et al. found in lung-lavaged rabbits less release of chemical inflammatory mediators in BALF and less lung injury in the rabbits ventilated with HFOV than ventilated with $\mathrm{CV}$ (31). In contrast, Thome et al. found no beneficial effect in patients that were treated with HFOV compared with those treated with conventional ventilation with respect to concentrations of albumin and pro-inflammatory cytokines in BALF (32). However it could be argued that in that study, the BALF collected during the first day was pooled and maybe differences between the two groups have disappeared already after 12 or more hours after birth. In this study, investigated parameters concerning clotting showed only for platelet function a statistically significant difference between HFOV and $\mathrm{CV}$, suggesting a protective effect of HFOV on the activation of clotting. Although no significant differences were found, the trend of aPTT and thrombin inhibition, as well as thrombin in BALF, further support this hypothesis.

\section{CONCLUSION}

We conclude that the signs of systemic activation of inflammation, especially activation of PMNs and release of $\beta$-glucuronidase can be found in the ventilated preterm lamb with RDS within 15 min after birth. Pooling of PMNs in the lungs leads to subsequent release of elastase. Afterward, or due to activation of inflammation, clotting is activated systemically as well as locally. The activation takes place in the lambs ventilated with $\mathrm{CV}$ as well as in the lambs ventilated with HFOV, but HFOV possibly attenuates activation. Further research directs to the very early, i.e. in the first 15 min after birth, activation 
of inflammation. Once we have more insight in the initial steps in the cascade, therapeutic interventions, for example surfactant instillation, can be evaluated.

\section{REFERENCES}

1. Merritt TA, Cochrane CG, Holcomb K, Bohl B, Hallman M, Strayer D, Edwards DK, Gluck L 1983 Elastase and alpha 1-proteinase inhibitor activity in tracheal aspirates during respiratory distress syndrome. Role of inflammation in the pathogenesis of bronchopulmonary dysplasia. J Clin Invest 72:656-666

2. Murch SH, Costeloe K, Klein NJ, Rees H, McIntosh N, Keeling JW, MacDonald TT 1996 Mucosal tumor necrosis factor-alpha production and extensive disruption of sulfated glycosaminoglycans begin within hours of birth in neonatal respiratory distress syndrome. Pediatr Res 40:484-489

3. Merritt TA, Puccia JM, Stuard ID 1981 Cytologic evaluation of pulmonary effluent in neonates with respiratory distress syndrome and bronchopulmonary dysplasia. Acta Cytol 25:631-639

4. Buron E, Garrote JA, Arranz E, Oyaguez P, Fernandez CJ, Blanco QA 1999 Markers of pulmonary inflammation in tracheobronchial fluid of premature infants with respiratory distress syndrome. Allergol Immunopathol Madr 27:11-17

5. Little S, Dean T, Bevin S, Hall M, Ashton M, Church M, Warner J, Shute J 1995 Role of elevated plasma soluble ICAM-1 and bronchial lavage fluid IL-8 levels as markers of chronic lung disease in premature infants. Thorax 50:1073-1079

6. Groneck P, Gotze Speer B, Oppermann M, Eiffert H, Speer CP 1994 Association of pulmonary inflammation and increased microvascular permeability during the development of bronchopulmonary dysplasia: a sequential analysis of inflammatory mediators in respiratory fluids of high-risk preterm neonates. Pediatrics 93:712-718

7. Ogden BE, Murphy SA, Saunders GC, Pathak D, Johnson JD 1984 Neonatal lung neutrophils and elastase/proteinase inhibitor imbalance. Am Rev Respir Dis 130:817821

8. Speer CP 1999 Inflammatory mechanisms in neonatal chronic lung disease. Eur J Pediatr 158 Suppl. 1:S18-S22

9. Bengtsson A 1993 Cascade system activation in shock: Acta Anaesthesiol Scand Suppl 98:7-10

10. Brus F, van Oeveren W, Okken A, Oetomo SB 1997 Number and activation of circulating polymorphonuclear leukocytes and platelets are associated with neonatal respiratory distress syndrome severity. Pediatrics 99:672-680

11. Brus F, van Oeveren W, Okken A, Oetomo SB 1994 Activation of the plasma clotting, fibrinolytic, and kinin- kallikrein system in preterm infants with severe idiopathic respiratory distress syndrome. Pediatr Res 36:647-653

12. Singhal KK, Parton LA 1996 Plasminogen activator activity in preterm infants with respiratory distress syndrome: relationship to the development of bronchopulmonary dysplasia. Pediatr Res 39:2:229-235

13. Chan AK, Berry L, Mitchell L, Baranowski B, O’Brodovich H, Andrew M 1998 Effect of a novel covalent antithrombin-heparin complex on thrombin generation on fetal distal lung epithelium. Am J Physiol 274:L914-L921

14. Schmidt BK 1994 Antithrombin III deficiency in neonatal respiratory distress syndrome. Blood Coagul Fibrinolysis 5(suppl 1):S13-S17
15. Brus F, van Oeveren W, Okken A, Oetomo SB 1997 Disease severity is correlated with plasma clotting and fibrinolytic and kinin-kallikrein activity in neonatal respiratory distress syndrome. Pediatr Res 41:120-127

16. Brus F, van Oeveren W, Heikamp A, Okken A, Oetomo SB 1996 Leakage of protein into lungs of preterm ventilated rabbits is correlated with activation of clotting, complement, and polymorphonuclear leukocytes in plasma. Pediatr Res 39:958-965

17. Kawano T, Mori S, Cybulsky M, Burger R, Ballin A, Cutz E, Bryan AC 1987 Effect of granulocyte depletion in a ventilated surfactant-depleted lung. J Appl Physiol $62: 27-33$

18. HiFO Study Group 1993 Randomized study of high-frequency oscillatory ventilation in infants with severe respiratory distress syndrome. J Pediatr 122:609-619

19. Clark RH, Gerstmann DR, Null-DM J, deLemos RA 1992 Prospective randomized comparison of high-frequency oscillatory and conventional ventilation in respiratory distress syndrome. Pediatrics 89:5-12

20. Jobe AH, Ikegami M, Jacobs HC, Jones SJ 1983 Surfactant pool sizes and severity of respiratory distress syndrome in prematurely delivered lambs. Am Rev Respir Dis 127:751-755

21. Platts-Mills TAE 1974 Activation of the alternative pathway of human complement by rabbit cells. J Immunol 113:348-358

22. Baehner RL 1975 Subcellular distribution of nitroblue tetrazolium reductase (NBT-R) in human polymorphonuclear leukocytes (PMN). J Lab Clin Med 86:785-792

23. Heilmann EJ, Kundu SK, Sio R, Garcia C, Gomez R, Christie DJ 1997 Comparison of four commercial citrate blood collection systems for platelet function analysis by the PFA-100 system. Thromb Res 87:159-164

24. Marsh DJ, Frasier C, Decter J 1965 Measurement of urea concentration in nanoliter specimens of renal tubular fluid and capillary blood. Annal Biochem 11:73-80

25. Lowry OH, Rosenbrough NJ, Farr AL, Randall RJ 1951 Protein measurement with the Folin phenol reagent. J Biol Chem 193:265-275

26. Lengas A, Poletti V, Pacifico L, di Domizio C, Patelli M, Spiga L 1994 Acute lung inflammation: neutrophil elastase versus neutrophils in the bronchoalveolar lavageneutrophil elastase reflects better inflammatory intensity. Intensive Care Med 20:354359

27. Brus F, van Oeveren W, Okken A, Bambang SO 1996 Activation of circulating polymorphonuclear leukocytes in preterm infants with severe idiopathic respiratory distress syndrome. Pediatr Res 39:456-463

28. Wagner MH, Sonntag J, Strauss E, Obladen M 1999 Complement and contact activation related to surfactant response in respiratory distress syndrome. Pediatr Res 45:14-18

29. Carlton DP, Albertine KH, Cho SC, Lont M, Bland RD 1997 Role of neutrophils in lung vascular injury and edema after premature birth in lambs. J Appl Physiol 83:1307-1317

30. Mannaioni PF, Di Bello MG, Masini E 1997 Platelets and inflammation: role of platelet-derived growth factor, adhesion molecules and histamine. Inflamm Res 46:4-18

31. Imai Y, Kawano T, Miyasaka K, Takata M, Imai T, Okuyama K 1994 Inflammatory chemical mediators during conventional ventilation and during high frequency oscillatory ventilation. Am J Respir Crit Care Med 150:1550-1554

32. Thome U, Gotze SB, Speer CP, Pohlandt F 1998 Comparison of pulmonary inflammatory mediators in preterm infants treated with intermittent positive pressure ventilation or high frequency oscillatory ventilation. Pediatr Res 44:330-337 